\title{
RESEARCH
}

Open Access

\section{Thyroid involvement in Chanarin-Dorfman syndrome in adults in the largest series of patients carrying the same founder mutation in ABHD5 gene}

\author{
Nacim Louhichi ${ }^{1 *}$ (D, Emna Bahloul ${ }^{2}$, Slaheddine Marrakchi ${ }^{2}$, Houda Ben Othman ${ }^{3}$, Chahnez Triki ${ }^{3}$,
} Kawthar Aloulou ${ }^{4}$, Lobna Trabelsi ${ }^{5}$, Nadia Mahfouth ${ }^{6}$, Zeineb Ayadi-Mnif ${ }^{7}$, Leila Keskes ${ }^{1}$, Faiza Fakhfakh ${ }^{8}$ and Hamida Turki ${ }^{2}$

\begin{abstract}
Background: Chanarin-Dorfman syndrome (CDS) is a rare syndromic disease related to an accumulation of triacylglycerol in most organs. The aim of our study was to investigate various organs in a large series of CDS patients.

Results: We report for the first time thyroid function impairment in CDS. Among 12 investigated patients, 7 showed thyroid function impairment. All of them were over 30 of age. The 5 remaining investigated patients with normal thyroid function were under 30. Thyroid loss of function is an unknown clinical feature of CDS that could gradually develop with age. Thyroid ultrasound showed an abnormal aspect in all investigated patients ( 6 with thyroid impairment and 3 with normal thyroid function). Cervical MRI done in 2 patients with thyroid impairment showed fat infiltration of thyroid parenchyma. Audiogram carried out in 8 of our patients showed sensorineural hearing impairment in all patients, although only 2 patients suffered from clinical hypoacusia. We also demonstrated that kidney could be a more commonly involved organ than previously reported in the literature. A poorly differentiated kidney parenchyma is a common feature in our series. One patient showed cerebellar atrophy and T2 hypersignal of brain's white matter in MRI. All patients carried the same founder mutation c.773(-1)G > A in the ABDH5 gene.

Discussion: Aside from the congenital ichthyosiform erythroderma, the most common symptom of CDS, in addition to other organs involvement frequently reported in the literature, we described thyroid dysfunction, an unreported feature, probably related to the lipid infiltration of the thyroid parenchyma. The association found between age and hypothyroidism in CDS patients could explain the gradually development of thyroid disease with age.
\end{abstract}

Conclusion: We reported a thyroid dysfunction and unreported ultrasonographic aspects of kidneys and cerebral MRI in CDS patients.

Methods: We performed clinical analyses in 15 patients in whom thyroid, liver, ocular, kidney, skeletal muscle and neurological involvement were explored. Genetic and molecular explorations were performed by direct sequence analysis. Software SPSS, Fisher's exact test and ANOVA were used for statistical analyses.

Keywords: Hypothyroidism, CDS, C.773(-1)G > A mutation, ABHD5 gene, Splice site

\footnotetext{
* Correspondence: nacim.louhichi@gmail.com

${ }^{1}$ Human Molecular Genetic Laboratory, Faculty of Medicine of Sfax, University

of Sfax, Sfax, Tunisia

Full list of author information is available at the end of the article
}

(c) The Author(s). 2019 Open Access This article is distributed under the terms of the Creative Commons Attribution 4.0 International License (http://creativecommons.org/licenses/by/4.0/), which permits unrestricted use, distribution, and reproduction in any medium, provided you give appropriate credit to the original author(s) and the source, provide a link to the Creative Commons license, and indicate if changes were made. The Creative Commons Public Domain Dedication waiver (http://creativecommons.org/publicdomain/zero/1.0/) applies to the data made available in this article, unless otherwise stated. 


\section{Introduction}

Chanarin-Dorfman syndrome (CDS, MIM \# 275630) (neutral lipid storage disease with ichthyosis) is a rare syndromic autosomal recessive disease related to an accumulation of triacylglycerol in most organs [1]. Congenital ichthyosiform erythroderma (CIE) is the symptom shared by most of the patients. The disease is characterized by intracellular lipid droplets in multiple organs. Extra-cutaneous manifestations variably include fatty liver, myopathy, cataracts, and a variety of neurologic symptoms, such as mental retardation $[2,3]$.

CDS is induced by mutations in ABHD5 [4], which encodes abhydrolase domain containing 5 (ABHD5), an activator of adipose triglyceride lipase, leading to accumulation of triglycerides [5]. The ABHD5 is located on chromosome 3 and encodes 7 exons. A wide variety of mutations has been found worldwide in patients diagnosed with CDS. The ABDH5 consists of 349 amino acids with a molecular mass of $\sim 39 \mathrm{kD}$ and can bind to lipid droplets and activate lipolysis, i.e. the hydrolysis of triacylglycerol (TAG).

Herein, we report the largest group ever described in the literature of CDS patients. All these patients had the same founder homozygous $A B D H 5$ splice site mutation, which resulted in the skipping of the entire exon 6. Clinical history of associated hypothyroidism in one of our patients prompted us to investigate thyroid function in several patients followed for CDS in our department, along with extensive clinical, biological and radiological investigations of other organs.

\section{Patients and methods}

\section{Patients and clinical investigation}

We retrospectively collected clinical data from 15 patients belonging to 12 families sharing the same ethnic background and originated from the same geographic area, Kerkennah islands $(20 \mathrm{~km}$ off the east Tunisian coast). Informed consent was obtained from patients in accordance with the Code of Ethics of the World Medical Association (Declaration of Helsinki). Consanguinity and endogamy are characteristics of the local population of the island (Additional file 1: Figure S1). Prospective biological and radiological investigations of thyroid gland were performed to complete initially collected data.

We investigated thyroid function by assessing both Thyroid Stimulating Hormone (TSH) and Free Thyroxine (FT4) values in 8 patients and only TSH in 4 patients. Anti-thyroid antibodies were investigated in 6 patients. Peripheral blood smears were performed in 8 cases searching for "Jordan's anomaly", liver function: Aspartate Transaminase (AST), Alanine Transaminase (ALT) and ALP (Alkaline Phosphatase) in 13 patients, Creatine Phosphokinase (CPK) in 4 patients and renal function in 13 patients. Ophthalmological investigations were performed in 10 patients, audiogram in 8 patients, abdominal ultrasound in 10 patients, thyroid ultrasound in 9 patients, cervical MRI (Magnetic Resonance Imaging) in 2 patients with hypothyroidism and neuromuscular biopsy in one patient. Cerebral MRI was performed in two patients and electromyography (EMG) in one patient. We also investigated thyroid function $[(\mathrm{TSH})$ and (FT4)] in 11 healthy relatives, as controls, belonging to 3 families of CDS patients with hypothyroidism.

\section{Mutation analysis for $A B H D 5$ gene}

Genetic and molecular explorations were carried out for 15 patients belonging to 12 nuclear families. The 7 exons and flanking intron regions of the ABHD5 were analyzed for mutations in the CDS patients by direct sequence analysis. Following DNA extraction, the coding regions and intron-exon boundaries of the $A B H D 5$ were amplified by polymerase chain reaction (PCR) using generated primers covering the entire coding region (Additional file 3: Table $\mathrm{S} 1$ in the supplementary appendix). PCR was carried out on $50 \mu \mathrm{l}$ volume samples, in a GeneAmp ${ }^{\circ}$ PCR system 9700 (Applied Biosystems, Foster City, CA, USA). Each PCR reaction contains $100 \mathrm{ng}$ of genomic DNA, $0.4 \mu \mathrm{M}$ of each primer, $0.2 \mathrm{mM}$ of dNTPs, $1 \mathrm{IU}$ of Amplitaq and 10X PCR Buffer II in a final concentration of $1 \times 1.5 \mathrm{mM}$ of $\mathrm{MgCl}_{2}$. The mixture was denatured during $10 \mathrm{~min}$ at $95^{\circ} \mathrm{C}$ and then followed by 35 cycles: denaturing at $95^{\circ} \mathrm{C}$ for $45 \mathrm{~s}$, annealing at 65/ $60^{\circ} \mathrm{C}$ for $45 \mathrm{~s}$ and an extension at $72^{\circ} \mathrm{C}$ for $45 \mathrm{~s}$; with a final extension at $72{ }^{\circ} \mathrm{C}$ for $7 \mathrm{~min}$. Thereafter, the purified amplicons were directly sequenced using a dye terminator cycle sequencing kit V1.1 with an ABI sequence analyser 3100 Avant (Applied Biosystems, Foster City, CA, USA) according to the manufacturer's recommendations.

\section{Statistical analysis}

The software SPSS for Windows (version 20.0) (IBM SPSS Inc.) was used for the statistical analysis. Fisher's exact test was used to study the association between thyroid involvement and CDS. ANOVA was used to compare occurrence of hypothyroidism in 2 age groups: 30 years or over and under 30. Statistical significance was defined as a $p$ value of less than 0.05 .

\section{Results}

Clinical features, cutaneous and extracutaneous findings in our patients are summarized in Table 1. The 


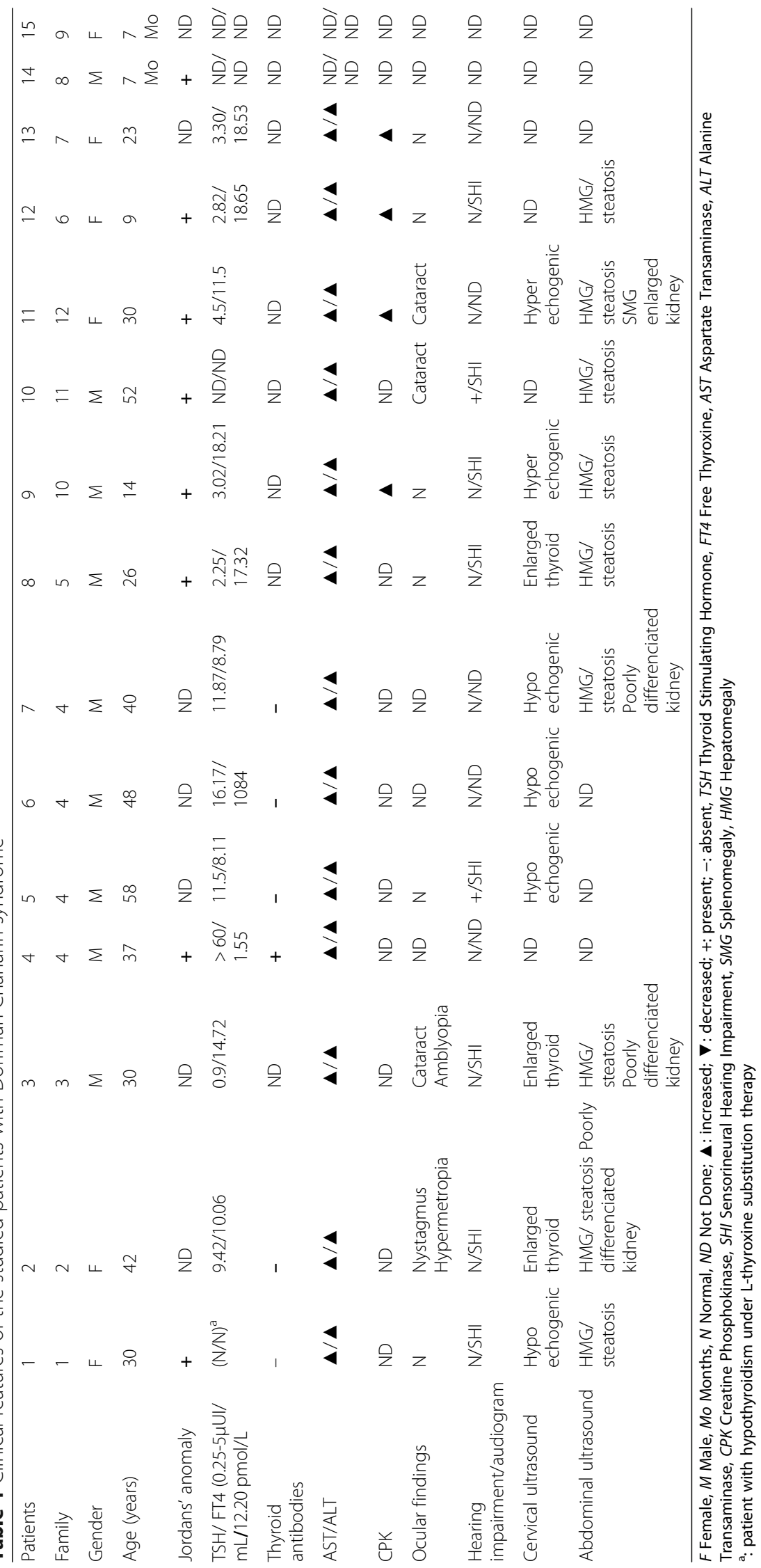




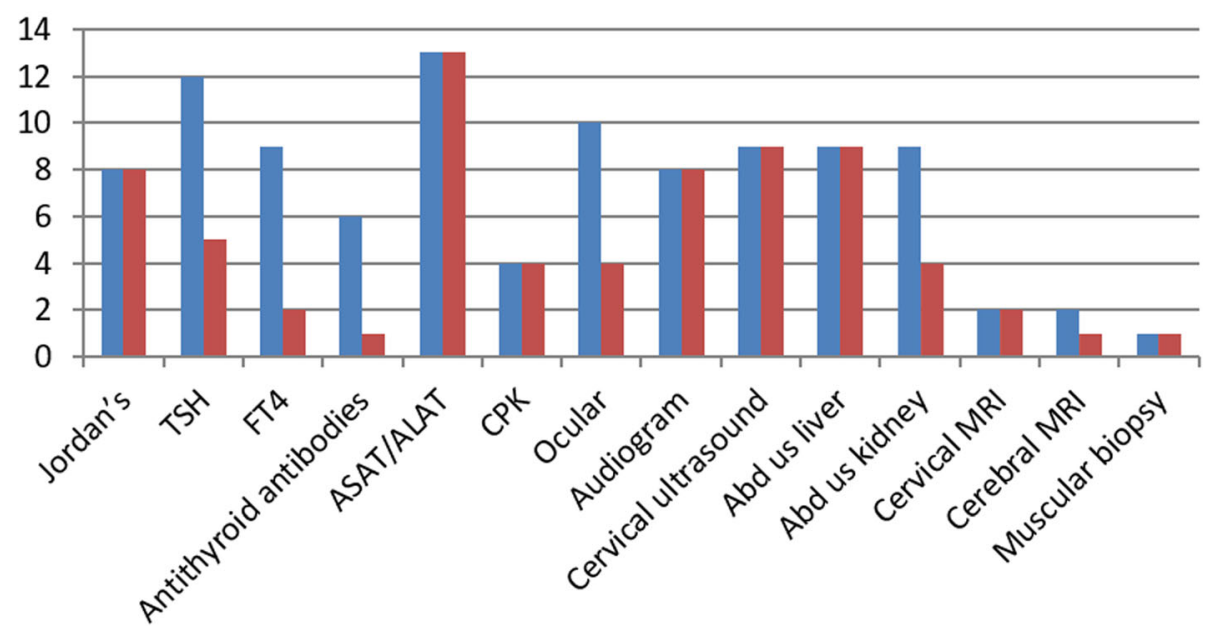

Fig. 1 Distribution of clinical, radiological data of patients for each analysis. Abdominal ultrasonography liver (Abd us liver), Abdominal ultrasonography kidney (Abd us kidney), Thyroid Stimulating Hormone (TSH), Free Thyroxine (FT4), Aspartate Transaminase (ASAT), Alanine Transaminase (ALAT), Creatine Phosphokinase (CPK), Magnetic Resonance Imaging (MRI)

distribution of clinical, radiological and biological data of patients for each analysis is summarized in Fig. 1.

\section{Clinical, laboratory and radiological data of CDS patients}

Nine males and 6 females aged between 7 months and 58 years were studied. Patients 4, 5, 6 and 7 were siblings. All patients presented with congenital ichthyosiform erythroderma. Eleven patients showed fine scales and 4 had large scales at the examination.

Cytoplasmic lipid droplets (Jordans'anomaly) in blood cells were found in the 8 investigated patients. Patients 14 and 15 , due to their young age (7 months) were only investigated for genetic variation.

\section{- Thyroid involvement}

Patient 1 was followed for hypothyroidism for many years before the diagnosis of CDS was made. Thyroid function was then investigated in 11 additional patients in our series. Thyroid function of patient 1 was normal under L-thyroxine substitution therapy. Seven patients among the 12 investigated subjects, aged 30 and over, had hypothyroidism. Only 5/12 patients, aged between 9 and 30, showed normal thyroid function. Significant association was found between age and hypothyroidism $(p=0.04)$.

Anti-thyroid antibodies investigated in 6 patients were elevated only in patient 4 who demonstrated the most severe thyroid function impairment.

Thyroid ultrasounds were carried out in 9 patients (6 patients with hypothyroidism and in 3 among the
5 patients with normal thyroid function) and showed abnormalities in all cases.

Among the 6 patients with hypothyroidism, 4 showed low echogenic aspect. In one patient, thyroid was enlarged with normal echogenic aspect and in the remaining patient thyroid size was normal but showed hyperechogenic aspect.

Among the 3 patients with normal thyroid function, 2 showed enlarged thyroid with normal structure and one hyperechogenic thyroid. However, thyroid ultrasound could not specifically show fatty infiltration of the thyroid.

To determine whether morphological and biological thyroid changes were related to fatty infiltration of the thyroid gland, cervical MRI was performed in 2 patients (1 and 4). It showed the same morphological aspect in the 2 investigated patients: In-Phase (IP) and Out-Of-Phase (OOP) sequences identified pathological fat infiltration of thyroid parenchyma by showing signal intensities drop on the OOP images comparatively to the IP images (Fig. 2).

Since patients are originated from an island with high degree of endogamy, fortuitous association between CDS and hypothyroidism could not be ruled out. Only sister of patient 1 was known to have hypothyroidism without CDS. She was supplemented with L-thyroxin therapy. Thus we investigated 10 additional healthy relatives (parents, siblings) from families 1, 2 and 4 and aged over 30 years. All showed normal TSH and FT4 values (Table 2). CDS group and healthy relatives were compared to see whether hypothyroidism was associated with CDS. Exact Fisher 

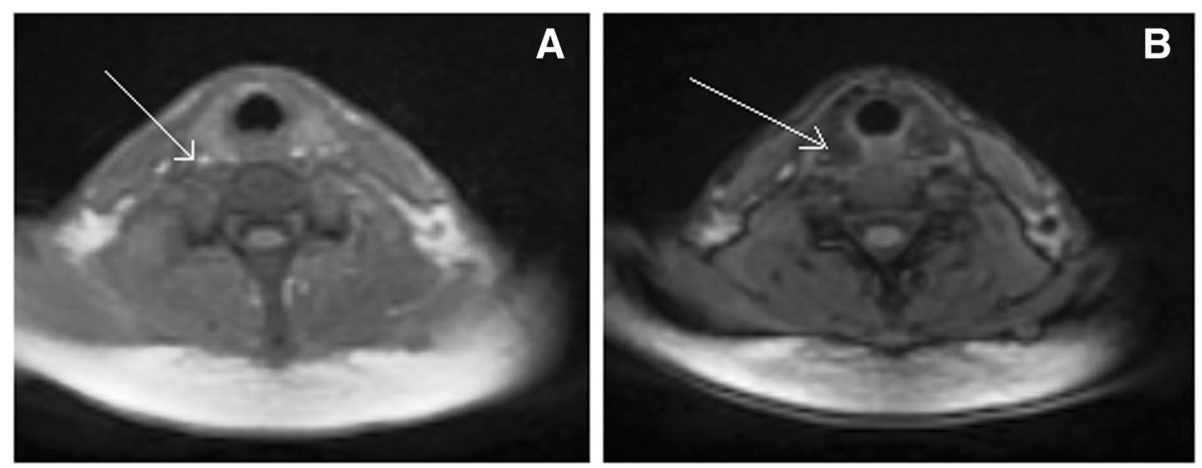

Fig. 2 Cervical MRI for patient 1 with In-Phase sequence (IP) (a) and Out-Of-Phase sequence (OOP) (b) showing drop of signal intensities. Cervical MRI for patient 1 shows lipid-rich thyroid gland. In-Phase sequence (IP) (a) demonstrates an intermediate signal (arrow). Out-of-phase sequence (b), thyroid gland shows a reduction of signal intensity (arrow), indicating that the gland is most likely infiltrated by lipids. This signal intensity difference is related to difference between water and fat protons resonance frequencies

test concluded to a statistically significant association between CDS and hypothyroidism ( $p=0.027)$.

As thyroid biopsy is an invasive investigation, we decided to not carry out such exploration in patients who carried impaired thyroid function.

\section{Neurological symptoms}

Patients 12 and 13 showed a slight mental retardation. Cerebral MRI was realized in both patients. Patient 12 with clinical microcephaly did not show any MRI abnormality. Patient 13 showed cerebellar atrophy and a T2 hyper signal of brain's white matter. Patient 11 had learning difficulties during her scholarship. Audiogram systematically realized in 8 patients demonstrated sensorineural hearing impairment in all cases, but, clinically, only 3 patients complained from hypoacusia.

Table 2 FT4 and TSH values in healthy relatives of family 1, 2 and 4

\begin{tabular}{ccll}
\hline Families & Age & FT4 $(9-20 \mathrm{pmol} / \mathrm{L})$ & TSH $(0.25-5 \mu \mathrm{lU} / \mathrm{mL})$ \\
\hline Family 1 & & & 1.63 \\
II.3 & 62 & 12.69 & 2.11 \\
II.4 & 56 & 12.76 & $\mathrm{~N}$ \\
III.2 & 29 & $\mathrm{~N}$ & \\
Family 2 & & & 1.69 \\
II.3 & 79 & 14.30 & 2.03 \\
II.4 & 69 & 17.36 & 1.75 \\
III.3 & 39 & 16.60 & 3.70 \\
III.4 & 34 & 17.45 & \\
Family 4 & & & 3.25 \\
III.2 & 75 & 11.16 & 4.79 \\
IV.6 & 45 & 10.42 & 3.00 \\
IV.7 & 51 & 12.00 & 3.81 \\
IV.8 & 55 & 11.75 & \\
\hline
\end{tabular}

${ }^{\mathrm{a}}$ Known hypothyroidism under L-thyroxine substitution therapy
- Kidney involvement

Renal function was normal in 13 investigated patients. However, during abdominal ultrasound carried out in 10 cases, patient 11 showed kidney enlargement with splenomegaly and patients $2,3,7$ and 8 showed a poorly differentiated kidney parenchyma, with increased echogenicity and absence of corticomedullary differenciation. This aspect is not specific but could be related to lipid infiltration without biological expression.

- Liver involvement

Thirteen patients were investigated for hepatic function. All of them showed cytolysis.

Nine patients undergoing abdominal ultrasound, showed liver steatosis without cirrhosis or portal hypertension aspects. No liver biopsies were realized in our patients.

- Ocular signs and symptoms

Among 10 patients who underwent ophthalmological examination, 4, aged between 30 and 52, showed ocular anomalies (ectropion in 4 cases associated to cataract in 3 cases)

- Skeletal muscle manifestations

CPK was elevated in the 4 investigated patients. Neuromuscular biopsy in patient 12 showed muscle fatty infiltration with vacuoles in the type I fibers, although EMG was normal in the patient.

\section{Molecular findings}

Mutational screening of the $A B H D 5$ coding region and intron-exon boundaries revealed the common 
homozygous c.773(-1)G $>$ A mutation in the acceptor splice site of exon 6 in all explored patients (Additional file 2: Figure S2).

\section{Discussion}

We described 15 patients with neutral lipid storage disease with ichthyosis. We are focusing on the relevance of the newly described features of CDS.

\section{- Thyroid involvement}

The high rate of hypothyroidism in our series, mainly in patients aged over 30 , the exclusion of the autoimmune cause as well as the MRI aspect in 2 patients showing fatty infiltration of the thyroid, and the normal thyroid function in healthy relatives, support the hypothesis that lipid infiltration of thyroid in CDS may lead to thyroid impaired function.

Lipotoxicity was suspected to be a risk factor of subclinical hypothyroidism and thyroid gland among targeted organs affected by lipotoxicity [6]. In a cohort of 24,100 subjects, the risk of subclinical hypothyroidism was found to be positively associated with hypertriglyceridemia [6].

In an experimental study, it was demonstrated that the in vitro thyrocytes stimulation by palmitic acid, led to a dose dependent intracellular accumulation of triglycerides and free fatty acids and to a decreased levels of thyroglobulin, the precursor of thyroid hormones, sodium iodide and thyroperoxidase [7]. Thus, accumulation of triglycerides might lead to impaired thyroid hormones synthesis.

Thyroid function was investigated in a few CDS patients in the literature, aged between 7 months and 26 years old [8-11]. The thyroid function was normal and no thyroid ultrasound or MRI was performed [8-11]. The discrepancy with our series could be related to the young age of investigated patients in the literature. Moreover, given the significant association found between age and hypothyroidism in CDS patients, it may be thought that thyroid disease develops gradually with age in the CDS.

Our patients are originated from the same geographic area, Kerkennah. Epidemiological studies on hypothyroidism are lacking in Tunisia. Prevalence of subclinical hypothyroidism occurs in $4.3 \%$ in the US population [12]. However epidemiology could be different in Tunisia due to high degree of consanguinity in some areas of the country. Although there was a bias, in a Tunisian study of 10,848 patients in a department of endocrinology, it was showed that the most common form of thyroid disease was autoimmune thyroiditis: $14 \%$ of patients were diagnosed with thyroid disease and $9.9 \%$ with autoimmune thyroid disease, which is characterized by elevated antithyroid antibodies. The female predominance was also reported [13]. In our study, antithyroid antibodies were elevated only in one patient and we did not find a female predominance.

\section{Neurological symptoms}

Few reports of slight mental retardation (2 patients in our series), scholar learning difficulties (1 patient in our series), ataxia, hypotonia, epilepsy and microcephaly are found in the literature. The aspect of cerebellar atrophy and T2 hypersignal of brain's white matter seen in the MRI of patient 13, has never been described in the literature. Using MRI/MRS (MRS: Magnetic Resonance Spectroscopy), Huigen et al. found an abnormal signal at $1.3 \mathrm{ppm}$ in cerebral white matter and cortex, as well as basal ganglia, reflecting a widespread accumulation of lipids [14].

Hypoacusia or hearing loss was reported in 25 to $30 \%$ of cases in the literature but audiogram was not constantly realized [15]. It is known that hearing impairment can develop at any age and could be progressive. Although not life threatening disease, hearing loss is a condition that could lead to professional and social integration difficulties. So we recommend systematic hearing investigation in CDS.

\section{- Kidney involvement}

Only 3 cases of kidney involvement in CDS have been reported in the literature [16].Two of them presented with nephritic syndrome and in 2, kidney biopsy showed lipid vacuolization. In one case severity of the disease led to the death of the patient. The poorly differentiated kidney parenchyma and enlarged kidney has never been described in the literature. Probably a modifier gene could explain the involvement of kidney in very rare cases.

\section{- Liver involvement}

Liver function defects are the second most common feature reported in the literature. More than $60 \%$ of patients in previously reported series show either hepatosteatosis or liver cirrhosis which could be seen even in young age $[3,17]$. Evolution to cirrhosis was reported in a few cases. In our series we have no cirrhosis cases.

Steatosis, seen in the majority of our patients, could be explained by deficient cofactor activity in CDS leading to fatty acids deposition in various organs. Development of cirrhosis in some patients could be related to inflammation mediated by TNF $\alpha$ and IL-1 $\beta$. In CGI-58 knockout (LivKO) mice, enriched fatty acids intake leads to oxidative stress, increased serum aminotransferases and expression of mRNA of genes involved in inflammation (genes expressing TNF $\alpha$ and IL-1 $\beta)$ [18].

- Ocular signs and symptoms 
Most common ocular features, in our series, were cataract and ectropion. These two ocular signs are the most commonly reported in the literature, varying between 23 and 54\% [17]. Development of cataract could be related to age.

- Skeletal muscle manifestations

Muscular involvement was not extensively investigated in our series. Muscular involvement is a common feature of CDS frequently reported in the literature [17, 19]. Our findings were similar to previous reports.

\section{Molecular findings}

Molecular investigation of our patients showed the presence of the c.773(-1)G > A mutation. Sugiura K et al. explored the effect of this mutation on the aberrant cDNA and revealed the skipping of the entire exon 6 leading to premature translation termination [20]. The mutation has been reported only in Tunisian patients originated from two Tunisian islands (Djerba and Kerkennah) $[1,20]$.

These two islands can be considered as geographical isolates with confined populations, with strong endogamy and consanguinity [21]. We can consider the presence of a common ancestor for this founder mutation. Explorations of microsatellites markers for some patients confirmed this finding $[21]$.

\section{Conclusion}

Aside from the congenital ichthyosiform erythroderma, the most common symptom of CDS, in addition to other organs involvement, frequently reported in the literature, we described thyroid dysfunction, a previously unreported feature, probably related to the lipid infiltration of the thyroid parenchyma. Previously unreported ultrasonographic aspects of the kidneys and of cerebral MRI were also described. We also noted the frequency of audiogram's abnormalities in CDS patients that should be systematically performed.

\section{Additional files}

Additional file 1: Figure S1. Families' pedigrees (Families 8, 9 and 10 are nuclear families). (TIF $3412 \mathrm{~kb}$ )

Additional file 2: Figure S2. Sequence chromatograms of the $A B D H 5$ gene in the region of the $\mathrm{c} .773(-1) \mathrm{G}>\mathrm{A}$ mutation, showing a control, carrier and mutant subject. Nucleotide variations are underlined. (BMP $595 \mathrm{~kb}$ )

Additional file 3: Table S1. Primers used for the amplification of ABHD5 in patients with Dorfman-Chanarin syndrome. (DOCX 15 kb)

\section{Abbreviations}

ALP: Alkaline Phosphatase; ALT: Alanine Transaminase; AST: Aspartate Transaminase; CIE: Congenital ichthyosiform erythroderma; CPK: Creatine Phosphokinase; EMG: Electromyography; FT4: Free Thyroxine; HMG: Hepatomegaly; IP: In-Phase; MRI: Magnetic Resonance Imaging; MRS: Magnetic Resonance Spectroscopy; OOP: Out-Of-Phase;
SHI: Sensorineural hearing impairment; TAG: Triacylglycerol; TSH: Thyroid Stimulating Hormone

\section{Acknowledgements}

We thank all the patients for their cooperation in the present study. This work was supported in part by the Tunisian Ministry of Higher Education, Scientific Research and Technology.

\section{Funding}

No funding was received for this work.

All the material and data of the study are available upon request.

Availability of data and materials

Not applicable (Our manuscript does not contain any data which can not be shared).

\section{Authors' contributions}

NL: study concept and design, acquisition, analysis and interpretation of genetic and clinical data, manuscript elaboration. EB: acquisition of clinical data and manuscript elaboration and statistical analysis. SM: acquisition of clinical data and manuscript elaboration, and critical revision of manuscript for intellectual content. HBO: acquisition of clinical data. ChT: acquisition of clinical data. KA: acquisition of clinical data. LT: acquisition of clinical data. NM: acquisition of clinical data. ZAM: acquisition of clinical data. LK: critical revision of manuscript for intellectual content. FF: acquisition of genetic data and critical revision of manuscript for intellectual content. HT: acquisition of clinical data and critical revision of manuscript for intellectual content. All authors have read the manuscript and approved its submission and declare that there is no conflict of interest.

Ethics approval and consent to participate

Informed consent was obtained from patient in accordance with the Code of Ethics of the World Medical Association (Declaration of Helsinki).

Consent for publication

Written informed consent for publication of the MRI of Fig. 2 was obtained from patient.

The consent form is available to the Editor if requested.

\section{Competing interests}

The authors declare that they have no competing interests.

\section{Publisher's Note}

Springer Nature remains neutral with regard to jurisdictional claims in published maps and institutional affiliations.

\section{Author details}

${ }^{1}$ Human Molecular Genetic Laboratory, Faculty of Medicine of Sfax, University of Sfax, Sfax, Tunisia. ${ }^{2}$ Department of Dermatology, Hedi Chaker Hospital, Sfax, Tunisia. ${ }^{3}$ Department of Neuropediatric, Hedi Chaker Hospital, Sfax, Tunisia. ${ }^{4}$ Department of Ophthalmology, Centre Intermédiare, Sfax, Tunisia. ${ }^{5}$ Department of Endocrinology, Centre Intermédiare, Sfax, Tunisia. ${ }^{6}$ Department of Immunology, Hedi Chaker Hospital, Sfax, Tunisia. ${ }^{7}$ Department of Radiology, Hedi Chaker Hospital, Sfax, Tunisia. ${ }^{8}$ Molecular and Functional Genetics Laboratory, Department of Life Sciences, Faculty of Sciences of Sfax, University of Sfax, Sfax, Tunisia.

Received: 2 February 2019 Accepted: 10 May 2019

Published online: 22 May 2019

\section{References}

1. Bruno C, Bertini E, Di Rocco M, et al. Clinical and genetic characterization of Chanarin-Dorfman syndrome. Biochem Biophys Res Commun. 2008;369(4): 1125-8.

2. Williams ML, Koch TK, O'Donnell JJ, et al. Ichthyosis and neutral lipid storage disease. Am J Med Genet. 1985:20(4):711-26.

3. Peña-Penabad C, Almagro M, Martínez W, et al. Dorfman--Chanarin syndrome (neutral lipid storage disease): new clinical features. $\mathrm{Br} J$ Dermatol. 2001;144(2):430-2. 
4. Lefèvre $\mathrm{C}$, Jobard F, Caux F, et al. Mutations in CGI-58, the gene encoding a new protein of the esterase/lipase/thioesterase subfamily, in ChanarinDorfman syndrome. Am J Hum Genet. 2001;69(5):1002-12.

5. Lass A, Zimmermann R, Haemmerle G, et al. Adipose triglyceride lipasemediated lipolysis of cellular fat stores is activated by CGI-58 and defective in Chanarin-Dorfman syndrome. Cell Metab. 2006;3(5):309-19.

6. Zhao M, Tang X, Yang T, et al. Lipotoxicity, a potential risk factor for the increasing prevalence of subclinical hypothyroidism? J Clin Endocrinol Metab. 2015;100(5):1887-94.

7. Zhao M, Zhang X, Gao L, et al. Palmitic acid downregulates thyroglobulin (Tg), sodium iodide symporter (NIS), and Thyroperoxidase (TPO) in human primary Thyrocytes: a potential mechanism by which lipotoxicity affects thyroid? Int J Endocrinol. 2018;17:4215848

8. Aksu G, Kalkan Ucar S, Bulut Y, et al. Renal involvement as a rare complication of Dorfman-Chanarin syndrome: a case report. Pediatr Dermatol. 2008;25(3):326-31.

9. Cakmak E, Alagozlu H, Yonem O, et al. Steatohepatitis and liver cirrhosis in Chanarin-Dorfman syndrome with a new ABDH5 mutation. Clin Res Hepatol Gastroenterol. 2012;36(2):e34-7.

10. Srinivasan R, Hadzić N, Fischer J, Knisely AS. Steatohepatitis and unsuspected micronodular cirrhosis in Dorfman-Chanarin syndrome with documented ABHD5 mutation. J Pediatr. 2004;144(5):662-5.

11. Gupta N, Gothwal S, Satpathy AK, et al. Chanarin Dorfman syndrome: a case report with novel nonsense mutation. Gene. 2016;575(2 Pt1):359-62.

12. Hollowell JG, Staehling NW, Flanders WD, et al. Serum TSH, T(4), and thyroid antibodies in the United States population (1988 to 1994): National Health and nutrition examination survey (NHANES III). J Clin Endocrinol Metab. 2002;87(2):489-99.

13. Chabchoub G, Mnif M, Maalej A, et al. Epidemiologic study of autoimmune thyroid disease in South Tunisia. Ann Endocrinol. 2006;67(6):591-5.

14. Huigen MC, van der Graaf M, Morava E, et al. Cerebral lipid accumulation in Chanarin-Dorfman syndrome. Mol Genet Metab. 2015;114(1):51-4.

15. Redaelli C, Coleman RA, Moro L, et al. Clinical and genetic characterization of Chanarin-Dorfman syndrome patients: first report of large deletions in the ABHD5 gene. Orphanet J Rare Dis. 2010;5(1):33.

16. Verma SB, Mittal A, Wollina U, et al. Chanarin-Dorfman syndrome with rare renal involvement. $\mathrm{Br}$ J Dermatol. 2017:176(2):545-8.

17. Nur BG, Gencpinar P, Yuzbasıoglu A, et al. Chanarin-Dorfman syndrome: genotype-phenotype correlation. Eur J Med Genet. 2015;58(4):238-42.

18. Guo F, Ma Y, Kadegowda AK, et al. Deficiency of liver comparative gene Identification-58 causes steatohepatitis and fibrosis in mice. J Lipid Res. 2013;54(8):2109-20.

19. Barnérias C, Bassez G, Schischmanoff O. Chanarin-Dorfman syndrome in a 7year-old child: when myophathy and skin involvement are all but one. Med Sci(Paris). 2015;31 Spec No 3:11-13.

20. Sugiura K, Suga Y, Akiyama M. Dorfman-Chanarin syndrome without mental retardation caused by a homozygous ABHD5 splice site mutation that skips exon 6. J Dermatol Sci. 2014:75(3):199-201.

21. Romdhane L, Kefi R, Azaiez H, et al. Founder mutations in Tunisia: implications for diagnosis in North Africa and Middle East. Orphanet J Rare Dis. 2012;7:52

Ready to submit your research? Choose BMC and benefit from:

- fast, convenient online submission

- thorough peer review by experienced researchers in your field

- rapid publication on acceptance

- support for research data, including large and complex data types

- gold Open Access which fosters wider collaboration and increased citations

- maximum visibility for your research: over $100 \mathrm{M}$ website views per year

At $\mathrm{BMC}$, research is always in progress.

Learn more biomedcentral.com/submissions 